\title{
Microangiopathy of the brain and retina with hearing loss in a 50 year old woman: extending the spectrum of Susac's syndrome
}

\author{
R A Barker, J R Anderson, P Meyer, D J Dick, N J Scolding
}

\begin{abstract}
A 50 year old woman presented with a subacute onset of vertigo and diplopia followed by an encephalopathy with confusion, spasticity, ataxia, myoclonus, and multiple branch retinal arteriolar occlusions and unilateral sensorineural deafness. Brain biopsy confirmed multiple microinfarcts with no vasculitis. After the procedure she had a right iliofemoral deep vein thrombosis and was found to be heterozygous for the factor $V$ Leiden mutation. She was treated with anticoagulants and made a marked recovery with no relapses 6 months after presentation. This case extends the age range at which Susac's syndrome can present, and raises the possibility that the condition may be associated with abnormalities of coagulation. (F Neurol Neurosurg Psychiatry 1999;66:641-643)
\end{abstract}

Keywords: Susac's syndrome; activated protein C resistance

Neurology, Norfolk and Norwich Health

Care Trust, Brunswick

Road, Norwich,

Norfolk NR 1 3SR, UK

R A Barker

D J Dick

Department of

Pathology

J R Anderson

Department of Opthalmology

P Meyer

Department of Neurology,

Addenbrooke's

Hospital, Cambridge

CB 2 2QQ, UK

N J Scolding

Correspondence to: Dr Roger Barker,

Department of Neurology,

Addenbrooke's Hospital,

Cambridge CB 2 2QQ, UK.

Telephone 004401223

245151; fax 004401223

33694 .

Received 15 April 1998 and in revised form 26 June 1998 Accepted 29 June 1998 Susac et al first described a syndrome in 1979 in two female patients who presented with encephalopathy, retinal arteriolar branch occlusions, and deafness. Brain biopsy showed multifocal microinfarcts and it was suggested that the condition represented a microangiopathy of the brain, retina, and inner ear. ${ }^{1}$ Subsequently there have been 36 cases of Susac's syndrome reported in the literature ${ }^{2}$ and although the condition was initially thought to exclusively involve young women between the ages of 18 and 40 years of age, ${ }^{23}$ there have been reports of the condition affecting men. ${ }^{4-6}$ We now describe a 50 year old woman with a clinical picture typical of this disorder with histopathological confirmatory findings who went on to have an extensive iliofemoral deep vein thrombosis and was found to be heterozygous for the factor V Lei-

\section{Case history}

A 50 year old right handed woman presented at the end of August 1996 with a 3 day history of intermittent vertical diplopia followed by worsening vertigo, nausea, vomiting, and a low grade headache. Medical history was unre- markable. She had been a live kidney donor to her son in 1995. She smoked 20 cigarettes a day and had been on hormone replacement therapy for 4 years. Examination on admission showed her to have no systemic abnormalities and she was apyrexial. Neurological examination disclosed jerky nystagmus in all directions of gaze and an extensor right plantar response. Investigations showed a normal haematological and biochemical screen as well as MRI of her brain. Four days after admission she developed worsening dizziness, urinary frequency, and limb and truncal ataxia. She then became intermittently confused and drowsy and developed spasticity in the limbs with hyperreflexia and bilateral extensor plantar responses. She was given a 10 day course of acyclovir and thiamine without any improvement, but a 4 day course of intravenous methylprednisolone $(0.5$ $\mathrm{g}$ /day) followed by a reducing course of oral steroids (60 mg prednisolone) produced an appreciable but short lived improvement in her clinical condition.

Further investigations at this time continued den mutation. to be either normal or negative and included full blood count, erythrocyte sedimentation rate, urea and electrolytes, liver function tests, glucose, thyroid function tests, C reactive protein, serum angiotensin converting enzyme, calcium, phosphate, autoantibody screen including antinuclear antibodies, antineutrophil cytoplasmic antibodies and antineuronal antibodies. Viral, Lyme and an atypical pneumonia serological screen were all negative. Her CSF was analysed on three occasions and each time had less than 5 white cells $/ \mathrm{ml}$, but a raised protein up to $1.7 \mathrm{~g} / 1$ with a normal glucose and lactate concentration. Oligoclonal immunoglobulin bands were detected that were identical in the CSF and serum. Brain MRI was repeated and showed a solitary lesion on the T2 scan in the anterior portion of the left globus pallidus (figure A). Chest radiography and $\mathrm{CT}$ of the abdomen and pelvis and chest were all normal, as were her ECG, and transthoracic and transoesophageal echocardiogram. The normality of the echocardiogram test is important given the fact that there has been one reported case of Susac's syndrome with a patent foramen ovale. ${ }^{7}$ Bone marrow biopsy (aspirate and trephine) on two occa- 

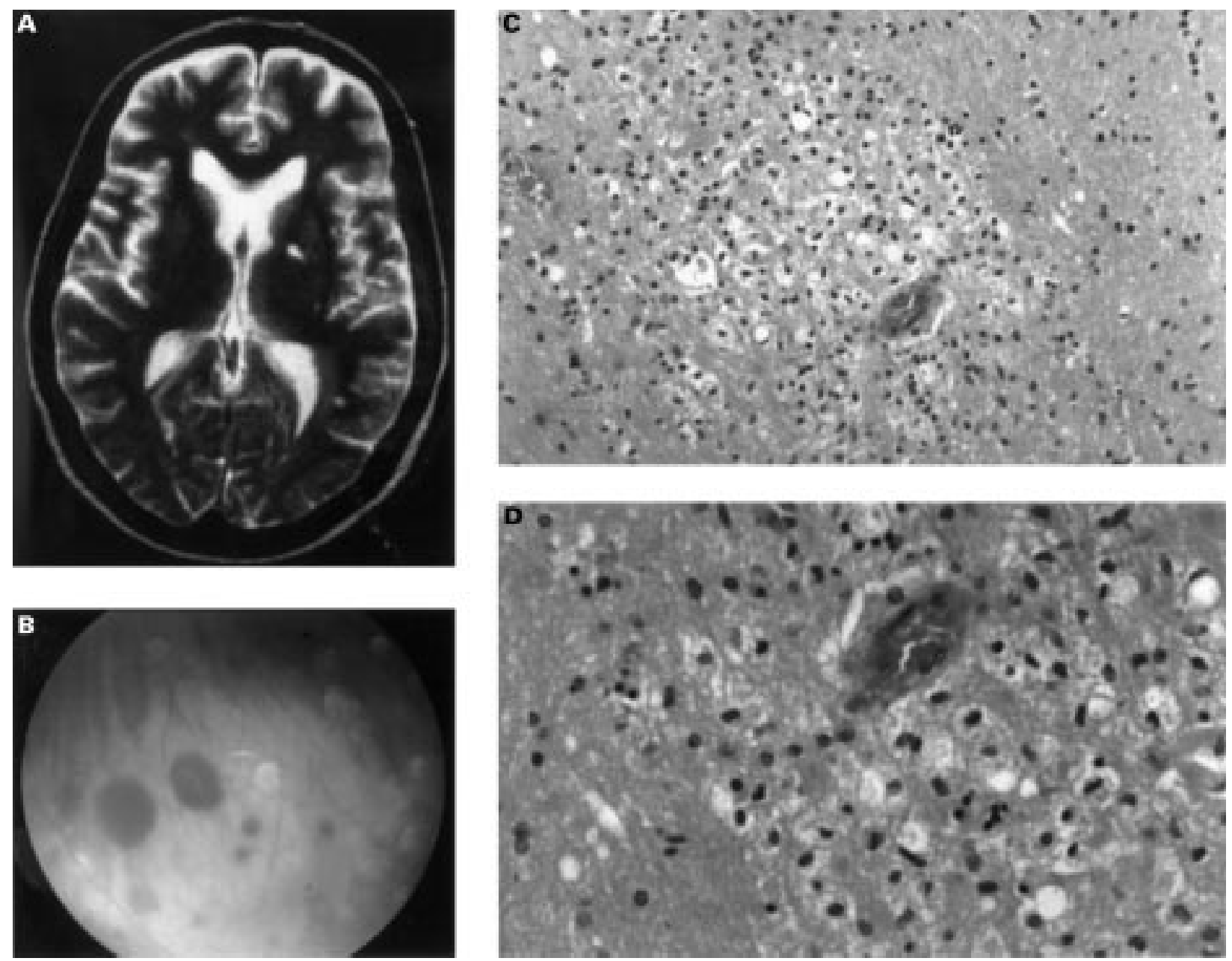

(A) T2 weighted MRI of the brain showing the solitary lesion in the anterior portion of the left globus pallidus. (B) Multiple exudates and haemorrhages associated with the retinal microangiopathy. (C) Cerebral white matter showing pericapillary rarefaction and infiltration by foamy macrophages that have engulfed lipid material derived from tissue necrosis (haematoxylin and eosin). (D) Detail of (C) showing pericapillary foamy macrophages but no vascular abnormality. The capillary lumen is patent and there is no evidence of vasculitis (haematoxylin and eosin).

sions showed only non-specific reactive changes. Her EEG showed diffuse slowing bilaterally and cerebral angiography was unremarkable. Opthalmological review, however, showed retinal arteriolar narrowing with some occlusions and infarcts (figure B) and on audiometry she had a right sided sensorineural deafness to all frequencies although both her visual evoked responses and brain stem auditory evoked responses were normal.

In view of her continued deterioration and the development of generalised non-stimulus sensitive myoclonic jerks, a right frontal lobe burr hole biopsy was undertaken. Cerebral biopsy showed microinfracts of varying ages with tiny foci of eosinophilic ischaemic neurons in the cerebral cortex and perivascular rarefaction, breakdown of axons, and accumulation of foamy macrophages in the white matter (figure C, D). Four days after this biopsy, despite being on heparin prophylaxis, she developed a right iliofemoral vein thrombosis established by CT and was subsequently found to be heterozygous for the factor $\mathrm{V}$ Leiden mutation. The rest of her clotting screen was normal including the level of protein C and S activity. She was started on intravenous heparin and then oral warfarin, which she continues to take now 6 months after her deep vein thrombosis. At the time of this thrombosis she developed abnormal liver function tests which at their worst showed an alanine transaminase concentration of $141 \mathrm{U} / 1$, a $\gamma-\mathrm{GT}$ concentration of $275 \mathrm{U} / 1$, and an alkaline phosphatase concentration of $191 \mathrm{U} / 1$ but with a normal bilirubin. These have subsequently returned to normal.

Over the course of the next 3 months she slowly improved on no treatment other than the anticoagulants. She has some mild cognitive impairments with difficulty walking due to a combination of spasticity and ataxia but her arm function and eye movements are now normal. She still has evidence of previous retinal arteriolar occlusions, but no new lesions have developed over the past 3 months and her sensorineural deafness on the right remains unchanged. An MRI performed 2.5 months into her illnesss at the time of improvement disclosed a few scattered non-specific white lesions on the T2 scan with the previously noted lesion in the globus pallidus persisting.

\section{Discussion}

This woman fulfilled the criteria of Susac's syndrome as detailed in the original description by Susac in $1979 .{ }^{1}$ She had a microangio- 
pathy of the brain demonstrated on the brain biopsy together with multiple retinal arteriolar occlusions, and an asymmetric hearing loss. However, this patient was significantly older than the previous 36 cases in the literature ${ }^{2}$ and in addition had the added complication of a deep vein thrombosis, due in part to her being heterozygotic for the factor V Leiden mutation. Only one other case has had an associated clotting abnormality and that was in a patient with protein $\mathrm{S}$ deficiency. ${ }^{8}$

The diagnosis of Susac's syndrome (or the retinocochleocerebral syndrome) is made by recognition of the clinical triad and the presence of microangiopathic changes on brain biopsy. ${ }^{1-7}$ The hearing loss and visual disturbances are often asymptomatic in the encephalopathic stage, and can be highly asymmetric, as in our patient; and there are also reports of cases where not all of the clinical triad were found. ${ }^{23}$ By contrast, systemic features have only rarely been described in this condition ${ }^{9}$ and whereas our patient was not especially different in this respect, she did develop abnormal liver function tests as well as having oligoclonal bands in both the CSF and serum.

This case of Susac's syndrome is not only unusual in presenting at such a late age but also because she had an iliofemoral deep vein thrombosis and heterozygosity for the factor $\mathrm{V}$ Leiden mutation. Although this mutation is not uncommon, occurring in about $5 \%$ of the general population, ${ }^{10}$ it has only ever been associated with venous thromboses, ${ }^{11}$ and there are no published reports of an associated microvasculopathy. The relevance of this mutation to our patient's neurological presentation is unclear, but in the absence of any known aetiopathological process underlying this syndrome, such associations deserve further investigation.

In summary, we present a woman with the clinical and pathological features of Susac's syndrome. She was appreciably older than any of the previously reported cases and was also found to be heterozygotic for the factor V Leiden mutation which contributed to her having an iliofemoral deep vein thrombosis. The relevance of this mutation to the pathogenesis of Susac's syndrome, and the role of anticoagulation in its treatment remains unknown.

1 Susac JO, Hardimann JM, Selhorst JB. Microangiopathy of the brain and retina. Neurology 1979;29:313-6.

2 Papo T, Biousse V, Lehoang P, et al. Susac syndrome. Medicine 1998;77:3-11.

3 Susac JO. Susac's syndrome: the triad of microangiopathy of the brain and retina with hearing loss in young women. Neurology 1994;44:591-3.

4 Kaminska EA, Sadler M, Sangalang V, et al. Microangiopathic syndrome of encephalopathy, retinal vessel occlusion and hearing loss [abstract]. Can f Neurol Sci 1990;17:241. neuropsychological characteristics in a young man. Neurology 1996;47:266-8.

6 Vila N, Graus F, Blesa R, et al. Microangiopathy of the brain and retina (Susac's syndrome): two patients with atypical features. Neurology 1995;45:1225-6.

7 Gordon Dl, Hayreh SS, Adams HP Jr. Microangiopathy of the brain, retina and ear: improvement without immunosuppressive therapy. Stroke 1991;22:933-7.

8 Cafferty MS, Notis C, Kitei R, et al. Retinal artery occlusions, hearing loss, and stroke in a 19-year old abstract]. Neurology 1994;44:A267.

9 Nicolle MW, McLachlan RS. Microangiopathy with retinopathy, encephalopathy and deafness (RED-M) and systemic features. Semin Arthritis Rheum 1991;21:123-8.

10 Koster T, Rosendaal FR, de Ronde H, et al. Venous thrombosis due to poor anticoagulant response to activated pro1503-1506.

11 Koeleman BP, Reitsma PH, Bertina RM. Familial thrombophilia: a complex genetic disorder. Semin Hematol 1997;34:256-64. 